\title{
Endothelial heterogeneity and plasticity
}

\author{
Coert Margadant ${ }^{1}$
}

Published online: 20 May 2021

(C) The Author(s), under exclusive licence to Springer Nature B.V. 2021

\begin{abstract}
Vascular endothelial cells are highly plastic and show great phenotypic heterogeneity. In recent years, emerging technologies have identified a range of novel endothelial phenotypes and functions. In this Special Issue of Angiogenesis, we present a series of papers from leading experts in the field, highlighting the heterogeneity and plasticity of endothelial cells in health and disease.
\end{abstract}

Endothelial cells form the inner lining of our vessels and regulate the exchange of fluids, proteins, and cells between the circulation and surrounding tissues. While it is known for long that there are considerable differences between endothelial cells from lymphatic and blood vessels, as well as from arteries, veins, and capillaries, the full repertoire of endothelial phenotypic diversity is only beginning to be elucidated. Recent years have seen a surge into the identification of spatiotemporal molecular and functional heterogeneity of endothelial cells, supported by technologies such as single-cell RNAseq, lineage tracing, and intersectional genetics [1]. Together, these techniques have spurred the generation of epigenomic, transcriptomic, and proteomic signatures of endothelial cells [1-3]. Indeed, it is now clear that endothelial cells across organs and in different vascular beds, but even within the same vessel, have unique molecular identities, and employ unique molecular mechanisms to fulfill highly specialized needs [4].

In addition to these emerging insights into endothelial cell heterogeneity, it is becoming increasingly apparent that endothelial cells are remarkably plastic, and can adopt different phenotypes and cell fate during development, tissue repair, or disease. This is illustrated by the specification of tip and stalk cell identities during angiogenesis, or the specification of endothelial cells into arterial, venous, or lymphatic lineages. Endothelial commitment to the

Coert Margadant

c.margadant@amsterdamumc.nl

1 Angiogenesis Laboratory, Department of Medical Oncology, Cancer Center Amsterdam, Amsterdam University Medical Center, Location VUmc, De Boelelaan 1117, 1081 HV Amsterdam, The Netherlands appropriate lineage is essential for normal vascular development, and dysregulation of this process can give rise to arteriovenous malformations such as those observed in Osler-Weber-Rendu disease/Hereditary Hemorrhagic Telangiectasia (HHT), lymphatic abnormalities, or cerebral cavernous malformations (CCM), defects for which currently no treatment exists other than alleviation of symptoms such as bleeding [5, 6]. Furthermore, endothelial plasticity plays an important role in pathological conditions, repair, and regeneration, in particular during processes such as the endothelial-to-mesenchymal transition (endoMT) [7, 8], or the endothelial-to-hematopoietic transition (EHT), resulting in the generation of hematopoietic cells and precursors from hemogenic endothelium [9]. Finally, new endothelial cells can be generated from circulating endothelial colony forming cells (ECFCs) both in adults as well as during embryonic development [10], thus constituting a powerful source for vascular repair and regenerative medicine approaches.

In this Special Issue of Angiogenesis, we have compiled a series of review papers from leading experts in the field, together covering a range of topics related to the heterogeneity and plasticity of endothelial cells. First, Canu and Ruhrberg (https://doi.org/10.1007/s10456-021-09783-9) discuss the origins of the hematopoietic system and the pathways that regulate hematopoietic specification, either from endothelial cells through EHT, or from hematopoietic stem cells. Marziano, Gael et al. (https://doi.org/10.1007/s10456021-09785-7) highlight current concepts in the specification of arterial, venous, capillary and lymphatic endothelial cell identities during vascular development. Furthermore, they discuss how defects in these processes cause vascular malformations, and potential therapeutic strategies for their treatment. Fernandez-Chacon et al. (https://doi.org/10.1007/ 
s10456-021-09793-7) revisit the role of the Notch signaling pathway in vascular biology and discuss recent technologies that have elucidated the differential effects of Notch signaling in endothelial growth, remodeling, and hemostasis. Greenspan and Weinstein (https://doi.org/10.1007/s10456020-09761-7) focus on the zebrafish as a model system to study developmental endothelial cell specification, as well as endothelial plasticity in tissue repair, revascularization, and disease. Jafree et al. (https://doi.org/10.1007/s10456-02109784-8) describe emerging insights into the mechanisms and cell lineages underlying the formation of lymphatic vessels, and the techniques to investigate them. Koch, Lee et al. (https://doi.org/10.1007/s10456-021-09780-y) comprehensively describe the structural and functional heterogeneity of liver sinusoidal endothelial cells, a prime example of organ-specific angiodiversity, and its functional consequences for liver function in health and disease. Finally, Pasut et al. (https://doi.org/10.1007/s10456-021-09797-3) discuss new endothelial cell subtypes and functions that have been identified recently by single-cell technologies. Moreover, they discuss distinct metabolic gene signatures in different endothelial subtypes, and highlight how these could be exploited therapeutically.

Besides these review papers, we also compile recent advances from Hong et al. (https://doi.org/10.1007/s10456020-09765-3), who show that vascular networks generated with human ECFCs are much better than networks with mature endothelial cells at supporting stem cell-derived cardiomyocyte function and engraftment, which is due to their constitutively high expression of the cardioprotective growth factor neuregulin-1. Mauri et al. (https://doi. org/10.1007/s10456-021-09774-w) report that the adaptor protein Grb2b is required for Vegfc/Vegfr3 signaling and the correct formation of the lymphatic vasculature in zebrafish, while formation of intersegmental veins is less dependent on Grb2b. Tang et al. (https://doi.org/10.1007/ s10456-020-09757-3) show that the targeted deletion of NADPH oxidase 4 in endothelial cells disrupts VEGF signaling and hypoxia-induced sprouting angiogenesis in mice, while in a clinical study, Kroon et al. (https://doi.org/10. 1007/s10456-020-09758-2) present evidence that the antifungal compound itraconazole, which inhibits VEGF interactions with VEGFR2, reduces the frequency and severity of bleeding episodes resulting from vascular malformations in HHT patients. Finally, Ruter et al. (https://doi.org/10. 1007/s10456-021-09777-7) describe a novel mechanism for the transition of angiogenic endothelial cells to a homeostatic and quiescent phenotype, dependent on SMAD6 and induced by blood flow-invoked shear stress.

Altogether, these papers describe key features of endothelial heterogeneity and plasticity, and highlight the importance of endothelial cell fate transitions for development and vascular function in health and disease. Future efforts will further illuminate the molecular mechanisms underlying endothelial heterogeneity and plasticity, and will establish the therapeutic potential of exploiting these processes for the treatment of disease, as well as for tissue repair and regeneration.

\section{References}

1. Kalucka J, de Rooij LPMH, Goveia J, Rohlenova K, Dumas SJ, Meta E, Conchinha NV, Taverna F, Teuwen LA, Veys K, GarcíaCaballero M, Khan S, Geldhof V, Sokol L, Chen R, Treps L, Borri M, de Zeeuw P, Dubois C, Karakach TK, Falkenberg KD, Parys M, Yin X, Vinckier S, Du Y, Fenton RA, Schoonjans L, Dewerchin M, Eelen G, Thienpont B, Lin L, Bolund L, Li X, Luo Y, Carmeliet $P$ (2020) Single-cell transcriptome atlas of murine endothelial cells. Cell 180(4):764-779

2. Paik DT, Tian L, Williams IM, Rhee S, Zhang H, Liu C, Mishra R, Wu SM, Red-Horse K, Wu JC (2020) Single-cell RNA sequencing unveils unique transcriptomic signatures of organ-specific endothelial cells. Circulation 142(19): 1848-1862

3. Tacconi C, He Y, Ducoli L, Detmar M (2021) Epigenetic regulation of the lineage specificity of primary human dermal lymphatic and blood vascular endothelial cells. Angiogenesis 24(1):67-82

4. Jakab M, Augustin HG (2020) Understanding angiodiversity: insights from single cell biology. Development 147(15):146621

5. Detter MR, Shenkar R, Benavides CR, Neilson CA, Moore T, Lightle R, Hobson N, Shen L, Cao Y, Girard R, Zhang D, Griffin E, Gallione CJ, Awad IA, Marchuk DA (2020) Novel murine models of cerebral cavernous malformations. Angiogenesis 23(4):651-666

6. Faughnan ME, Gossage JR, Chakinala MM, Oh SP, Kasthuri R, Hughes CCW, McWilliams JP, Parambil JG, Vozoris N, Donaldson J, Paul G, Berry P, Sprecher DL (2019) Pazopanib may reduce bleeding in hereditary hemorrhagic telangiectasia. Angiogenesis 22(1):145-155

7. Dejana E, Hirschi KK, Simons M (2017) The molecular basis of endothelial cell plasticity. Nat Commun 8:14361

8. Man S, Sanchez Duffhues G, Ten Dijke P, Baker D (2019) The therapeutic potential of targeting the endothelial-to-mesenchymal transition. Angiogenesis 22(1):3-13

9. Zhu Q, Gao P, Tober J, Bennett L, Chen C, Uzun Y, Li Y, Howell ED, Mumau M, Yu W, He B, Speck NA, Tan K (2021) Developmental trajectory of prehematopoietic stem cell formation from endothelium. Blood 136(7):845-856

10. Díaz Del Moral S, Barrena S, Muñoz-Chápuli R, Carmona R (2020) Embryonic circulating endothelial progenitor cells. Angiogenesis 23(4):531-541

Publisher's Note Springer Nature remains neutral with regard to jurisdictional claims in published maps and institutional affiliations. 